\title{
Photoactivation Analysis of Mineral Trioxide Aggregate: Preliminary Results
}

\author{
C. $\operatorname{EKE}^{a, b, *}, \mathrm{~K} . \mathrm{ER}^{c}$, C. SeGEBADE ${ }^{a}$ AND I. BozTOSUN ${ }^{d}$ \\ ${ }^{a}$ Akdeniz University, Nuclear Sciences Application and Research Center, Antalya, Turkey \\ ${ }^{b}$ Akdeniz University, Faculty of Education, Department of Mathematics and Science Education, \\ Division of Physics Education, Antalya, Turkey \\ ${ }^{c}$ Akdeniz University, Faculty of Dentistry, Department of Endodontics, Antalya, Turkey \\ ${ }^{d}$ Akdeniz University, Faculty of Science, Department of Physics, Antalya, Turkey
}

\begin{abstract}
The purpose of this study is to qualitatively analyse the elemental components of a mineral trioxide aggregate material using photoactivation. The material was irradiated for two hours using bremsstrahlung photons with an end-point energy of $18 \mathrm{MeV}$ from a clinical electron linear accelerator. After irradiation the gamma-ray emission of the sample was counted by gamma spectrometry using a high-purity germanium (HPGe) detector. The gamma-ray spectra were analyzed by computer software. Major and trace elements were detected.
\end{abstract}

DOI: 10.12693 /APhysPolA.132.869

PACS/topics: 25.20.-x, 29.20.Ej

\section{Introduction}

Mineral trioxide aggregate (MTA) is a root canal repair material for endodontics. The composition of MTAs are somewhat similar to that of Portland cement. Two different kinds of MTA are available, namely gray MTA and white MTA. Gray MTA was introduced in 1998, whilst white MTA was commercially available since 2002. The contents of iron and other metallic elements in white MTA is lower than those in gray MTA. Thus, they have different therapeutic effects concerning perforation repair, leakage outcomes, root-end filling, resorptive defects, direct pulp capping, and apexification [1-6].

The chemical composition of MTA has been investigated using different atomic and nuclear techniques (energy dispersive X-ray fluorescence spectroscopy (EDXRF), atomic absorption spectroscopy (AAS), X-ray diffraction spectroscopy (XRD), inductively coupled plasma atomic emission spectroscopy (ICP-AES), inductively coupled plasma atomic optical spectroscopy (ICP-OES) etc.) [5, 7-10]. However, usage of photoactivation analysis (PAA) for analytical studies of MTA has not been reported in the literature.

PAA has been used for elemental analysis of different types of materials. PAA has a great potential for instrumental multi-element analysis [11]. The detailed principles, applications and general features of PAA have been described elsewhere [12]. PAA allows to determine trace elements in geological, biological, and environmental material $[13,14]$. Using PAA, many elements can be analysed with higher accuracy and sensitivity than achievable with several other standard techniques. Analyses can be carried out instrumentally and in many cases nondestructively. Normally the procedure is uncomplicated and moderately time-consuming [15].

One purpose of this study is to demonstrate, by this example of application, the usability of a medical electron linear accelerators for PAA. Detailed descriptions of this machine can be found in $[16,17]$

\section{Material and method}

In this study, the MTA sample mass was $140 \mathrm{mg}$. It was placed about $17 \mathrm{~cm}$ downstream from the tungsten converter. The clinical electron linear accelerator has been modified to achieve a close exposure distance to the converter. The sample was irradiated with $18 \mathrm{MeV}$ bremsstrahlung photons. The exposure period was two hours.

For gamma-ray spectroscopy a coaxial n-type liquid nitrogen-cooled ORTEC HPGe detector was used. After a cooling time of two hours the MTA sample was placed in front of the detector head at a distance of $5 \mathrm{~cm}$. The total counting time was 86360 seconds (about 1 day). During the collection of the spectrum, intermediate spectra ("time stamps") were recorded without interruption of the counting. During the first 21600 seconds (about $6 \mathrm{~h}$ ) for short lived radio-nuclides, time stamps were recorded in steps of 1800 seconds (12 spectra). Afterwards, time stamps were recorded during 86400 seconds for long lived radio-nuclides in steps of 3600 seconds (24 spectra). Spectra were collected using MAESTRO-32 software [18].

\section{Results}

Obtained spectra were analysed using Gamma-W software [19]. A gamma-ray spectrum is shown in Fig. 1. 


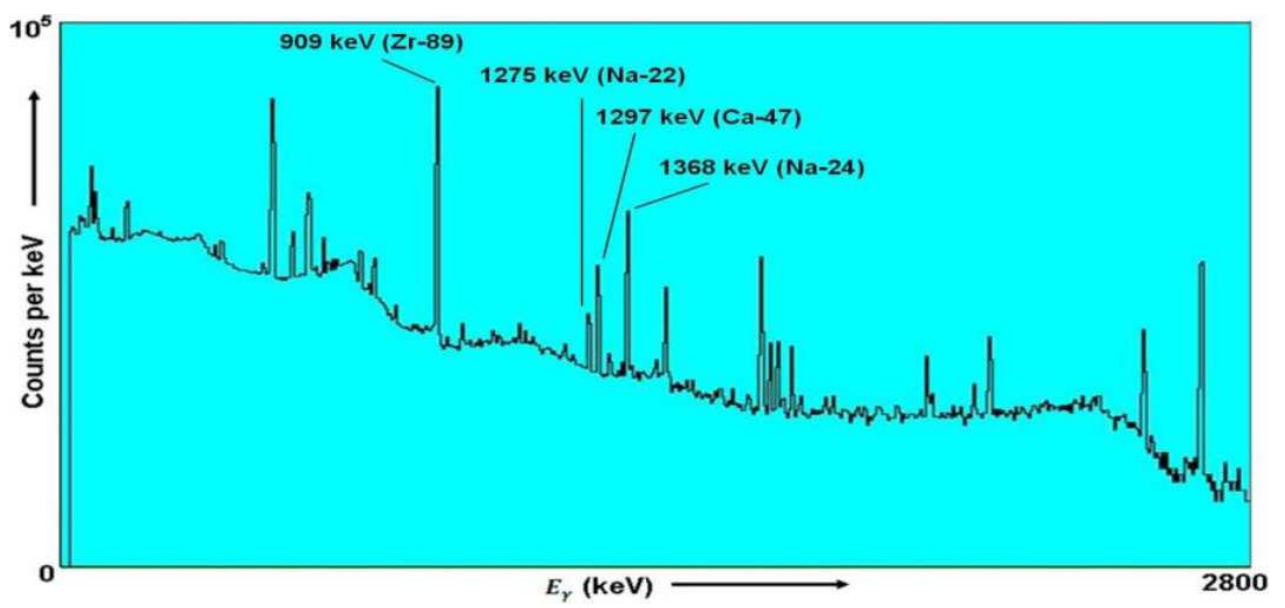

Fig. 1. Gamma-ray spectrum of a mineral trioxide aggregate after bremsstrahlung irradiation.

Detected reactions are compiled in Table I.

TABLE I

Qualitative instrumental photoactivation analysis of mineral trioxide aggregate components.

\begin{tabular}{|c|c|c|c|c|}
\hline Element & $\begin{array}{l}\text { Content } \\
+ \text { major } \\
- \text { trace }\end{array}$ & $\begin{array}{l}\text { Detected } \\
\text { reaction }\end{array}$ & $\begin{array}{l}\text { Radio- } \\
\text { nuclide }\end{array}$ & $\begin{array}{c}\text { Energy } \\
{[\mathrm{keV}][20]}\end{array}$ \\
\hline Uranium & - & ${ }^{238} \mathrm{U}(\gamma, \mathrm{n}){ }^{237} \mathrm{U}$ & ${ }^{237} \mathrm{U}$ & $59.5409 \pm 0.0001$ \\
\hline Zinc & - & ${ }^{68} \mathrm{Zn}(\gamma, \mathrm{p}){ }^{67} \mathrm{Cu}$ & ${ }^{67} \mathrm{Cu}$ & $\begin{array}{l}93.311 \pm 0.005 \\
184.577 \pm 0.01\end{array}$ \\
\hline $\begin{array}{c}\text { Calcium } \\
\text { Titanium }\end{array}$ & $\begin{array}{l}+ \\
- \\
\end{array}$ & $\begin{array}{c}{ }^{48} \mathrm{Ca}(\gamma, \mathrm{n})^{47} \mathrm{Ca} \\
{ }^{48} \mathrm{Ti}(\gamma, \mathrm{p})^{47} \mathrm{Sc}\end{array}$ & ${ }^{47} \mathrm{Sc}$ & $159.381 \pm 0.015$ \\
\hline Calcium & + & $\begin{array}{l}{ }^{48} \mathrm{Ca}(\gamma, \mathrm{n}){ }^{47} \mathrm{Ca} \\
{ }^{44} \mathrm{Ca}(\gamma, \mathrm{p}){ }^{43} \mathrm{~K}\end{array}$ & $\begin{array}{l}{ }^{47} \mathrm{Ca} \\
{ }^{43} \mathrm{~K}\end{array}$ & \begin{tabular}{|c|}
$807.86 \pm 0.1$ \\
$1297.09 \pm 0.1$ \\
$372.760 \pm 0.007$ \\
$617.490 \pm 0.006$
\end{tabular} \\
\hline Bromine & - & ${ }^{79} \mathrm{Br}(\gamma, 2 \mathrm{n}){ }^{77} \mathrm{Br}$ & ${ }^{77} \mathrm{Br}$ & $238.98 \pm 0.07$ \\
\hline Strontium & + & \begin{tabular}{|l|}
${ }^{88} \mathrm{Sr}(\gamma, \mathrm{n}){ }^{87 m} \mathrm{Sr}$ \\
${ }^{87} \mathrm{Sr}(\gamma, \gamma \mathrm{x})^{87 m} \mathrm{Sr}$ \\
\end{tabular} & ${ }^{87 m} \mathrm{Sr}$ & $388.531 \pm 0.003$ \\
\hline Arsenic* & - & ${ }^{75} \mathrm{As}(\gamma, \mathrm{n}){ }^{74} \mathrm{As}$ & ${ }^{74} \mathrm{As}$ & $\begin{array}{l}595.83 \pm 0.08 \\
634.78 \pm 0.08 \\
\end{array}$ \\
\hline Zirconium & + & $\begin{array}{l}{ }^{90} \mathrm{Zr}(\gamma, \mathrm{n}){ }^{89} \mathrm{Zr} \\
{ }^{96} \mathrm{Zr}(\gamma, \mathrm{n}){ }^{95} \mathrm{Zr}\end{array}$ & $\begin{array}{l}{ }^{89} \mathrm{Zr} \\
{ }^{95} \mathrm{Zr}\end{array}$ & \begin{tabular}{|c|}
$909.15 \pm 0.15$ \\
$724.192 \pm 0.004$ \\
$756.725 \pm 0.012$ \\
\end{tabular} \\
\hline Sodium & + & ${ }^{23} \mathrm{Na}(\gamma, \mathrm{n})^{22} \mathrm{Na}$ & ${ }^{22} \mathrm{Na}$ & $1274.537 \pm 0.007$ \\
\hline Magnesium & + & ${ }^{25} \mathrm{Mg}(\gamma, \mathrm{p})^{24} \mathrm{Na}$ & ${ }^{24} \mathrm{Na}$ & $1368.626 \pm 0.005$ \\
\hline
\end{tabular}

* probably a trace contamination.

$(\gamma, \mathrm{n}),(\gamma, 2 \mathrm{n}),(\gamma, \mathrm{p})$ and $\left(\gamma, \gamma^{\prime}\right)$ reactions were detected in stable elements in MTA by PAA. $(\gamma, n)$ reaction is dominant because cross section of this reaction is higher than those of other reactions in giant dipole resonance (GDR) region [12]. $(\gamma, \mathrm{n})$ reaction was detected for ${ }^{238} \mathrm{U}$, ${ }^{48} \mathrm{Ca},{ }^{88} \mathrm{Sr},{ }^{75} \mathrm{As},{ }^{90} \mathrm{Zr},{ }^{96} \mathrm{Zr}$ and ${ }^{23} \mathrm{Na}$ elements. $(\gamma, 2 \mathrm{n})$ was detected for ${ }^{79} \mathrm{Br},(\gamma, \mathrm{p})$ reaction was detected for ${ }^{68} \mathrm{Zn},{ }^{48} \mathrm{Ti},{ }^{44} \mathrm{Ca}$ and ${ }^{25} \mathrm{Mg}$ elements. $(\gamma, \gamma)$ reaction was detected for ${ }^{87} \mathrm{Sr}$ element. Major (Ca, Sr, Zr, Na and $\mathrm{Mg}$ ) and trace (U, Zn, Ti, Br, As) elements were determined by qualitative spectrum analysis in MTA as shown in Table I.

\section{Conclusions}

This study gives information about elemental components of MTA, as preliminary result. Elemental components of medical materials can be defined by using photoactivation. The output energy and the beam power of a clinical electron linear accelerator suffice for photoactivation.

In a future study, using an appropriate calibration material, precise quantitative determinations will be carried out. Elemental components of white MTA and grey MTA can be compared quantitatively using photoactivation.

\section{Acknowledgments}

The authors gratefully acknowledge the support of the Scientific Research Projects Coordination Unit of Akdeniz University.

\section{References}

[1] S. Asgary, M. Parirokh, M.J. Eghbal, F. Brink, J. Endod. 31, 101 (2005).

[2] D.M. Ferris, J.C. Baumgartner, J. Endod. 30, 422 (2004).

[3] A.L. Perez, R. Spears, J.L. Gutmann, L.A. Opperman, Int. Endod. J. 36, 564 (2003).

[4] T.M. Bozeman, R.R. Lemon, P.D. Eleazer, J. Endod. 32, 425 (2006).

[5] T. Dammaschke, H.U.V. Gerth, H. Züchner, E. Schäfer, Dent. Mater. 21, 731 (2005).

[6] R.S. Schwartz, M. Mauger, D.J. Clement, W.A. III Walker, J. Am. Dent. Assoc. 130, 967 (1999).

[7] U.R. Funteas, J.A. Wallace, F.W. Fochtman, Aust. Endod. J. 29, 43 (2003).

[8] S. Asgary, M. Parirokh, M.J. Eghbal, S. Stowe, F. Brink, J. Mater. Sci.: Mater. Med. 17, 187 (2006).

[9] S.W. Chang, W.J. Shon, W. Lee, K.Y. Kum, S.H. Baek, K.S. Bae, Oral Surg. Oral Med. Oral Pathol. Oral Radiol. Endod. 109, 642 (2010). 
[10] M.C.G.O. Dorileo, M.C. Bandeca, F.L.M. Pedro, L.E.R. Volpato, O.Ag.Guedes, R.D. Villa, M.R. Tonetto, A.H. Borges, Sci. World. J. 2014, 983728 (2014).

[11] A. Chattopadhyay, R.E. Jervis, Anal. Chem. 46, 1630 (1974)

[12] C. Segebade, H.P. Weise, G.J. Lutz, Photon Activation Analysis, Walter de Gruyter, Berlin 1988.

[13] K. Masumoto, T. Ohtsuki, Y. Miyamoto, J.H. Zaidi, A. Kajikawa, H. Haba, K. Sakamoto, J. Radioanal. Nucl. Chem. 239, 495 (1999).

[14] Z. Randa, J. Kucera, J. Mizera, J. Ferana, J. Radioanal. Nucl. Chem. 271, 589 (2007).

[15] J. Green, D. Wells, B. Benson, S. Zaijing, H. Maschner, AIP Conference Proceedings 1099, 919 (2009).
[16] C. Eke, I. Boztosun, H. Dapo, C. Segebade, E. Bayram, J. Radioanal. Nucl. Chem. 309, 79 (2016).

[17] Elekta Digital Accelerator, Technical Training Guide, unpublishled, 2003.

[18] Maestro-32, www.ortec-online.com/download/ MAESTRO.pdf, accessed 17 May 2016.

[19] GAMMA-W, www.westmeier.com 3gammawfeatures.htm, accessed 17 May 2016.

[20] Nudat, National Nuclear Data Center (NNDC), www.nndc.bnl.gov/nudat2, accessed 17 May 2016, Brookhaven National Laboratory. 